\title{
Radio Frequency Identification (RFID) enhanced Indoor Navigation Framework for Seniors
}

\author{
Charalampos Tsirmpas \\ Biomedical Engineering Laboratory, School of Electrical \\ and Computer Engineering, National Technical University \\ of Athens, \\ Athens, Greece \\ htsirbas@biomed.ntua.gr
}

\section{Alexander Rompas}

Biomedical Engineering Laboratory, School of Electrical and Computer Engineering, National Technical University of Athens,

Athens, Greece

arompas@biomed.ntua.gr

\author{
Athanasios Anastasiou \\ Biomedical Engineering Laboratory, School of Electrical \\ and Computer Engineering, National Technical University \\ of Athens, \\ Athens, Greece \\ aanastasiou@biomed.ntua.gr
}

\section{Dimitris Koutsouris}

Biomedical Engineering Laboratory, School of Electrical and Computer Engineering, National Technical University of Athens,

Athens, Greece

dkoutsou@biomed.ntua.gr

\begin{abstract}
One of the most challenging limitations for the elderly and/or the visually impaired people is the inability of selfnavigation in unfamiliar indoor environments. Numerous existing indoor navigation systems have been proposed in order to solve such problems. However, these papers do not adequately examine the effectiveness of the systems in real time trials and are faced with a variety of technical and usability problems. In our paper, we present a unique and elegant architecture of an indoor navigation system as an integrated and tested system in real time. This solution enables the localization of the user, as well as the navigation, considering scenarios that the user might follow and suggests guidelines for self- navigation.
\end{abstract}

Keywords- Radio Frequency Identification (RFID), Indoor Navigation Systems (INS), Visually Impaired, Ambient Assisted Living, Indoor Localization

\section{INTRODUCTION}

It is a fact that over 285 million people are visually impaired worldwide, of whom 39 million are blind. Based on data released by the World Health Organization $85 \%$ of people living in blindness are over 50 years old[1]. According to Prevent Blindness America (2013) the four leading eye diseases affecting Americans are age-related macular degeneration, Cataract, Diabetic Retinopathy and Glaucoma [2]. The majority of elderly people are not prescribed on a single medication but on several at the same time, which leads inevitably to severe side effects such as confusion and lack of decision making [3]. Consequently, it is mandatory for these people, special assistance and health status monitoring to be provided. Integrated systems such as the one proposed by Kyriacou [4], combined with low power protocols [5] and compression techniques [6], focus on home monitoring solutions by transmitting vital biosignals through GSM in order to deliver the required ambient assisted environment. Third age people are often faced with significant vision problems. In many cases, the symptoms become permanent, making guidance from another person a necessity [7]. Elderly people, whether visually impaired or lacking memory, have a difficult time acknowledging the location they are in, or how to arrive to the destination they desire. As mentioned above, many elderly people require consistent help and guidance not only in the outdoor environment, but also indoors. While outdoor navigation is well established and available at almost all weather conditions, indoor navigation still remains a technological field without effective integrated solutions. This paper presents an integrated localization and navigation system aiming to assist elderly and/or visually impaired people in indoor environment. The main objective of the system is to overcome the problems that arise in navigating indoors, specifically in terms of accuracy and dynamic adaptivity to different surroundings.

Over the last decade many interesting Information and Communications Technology (ICT) solutions and designs have been developed and can be grouped as: network based navigation which is based on networking technologies, and as autonomous user positioning. Systems using the network are based on technologies such as Bluetooth, Ultra Wide Band (UWB), Wi-Fi or Radio Frequency Identification (RFID). WiFi and UWB technologies have better position accuracy than Bluetooth and RFID technologies [8]. In the case of RFID and Bluetooth technology the main disadvantage is positioning accuracy. Even though, Bluetooth receivers are not commercially affordable, the technology is simple, compatible and short-range communication technology. Things are different in means of accuracy and cost when RFID technology is involved. The accuracy depends on the type of RFID reader, the amount of RFID tags, and the type of RFID tags being 
used, whether they are active or passive. Active tags have the tendency to cost more than passive tags, because of the built in battery they are embedded with.

Also, as mobile phones supporting Near Field Technology (NFC) get widely known, applications that use mobiles for an assistance terminal increase rapidly. Recently, Ivanov [9] used a combination of RFID tags located on the doors of the rooms (two types of tags: navi tags and audio tags) to automatically activate an application on the mobile when the last one comes close to the tags. The application though can be installed only on mobile terminals that support profile MIDP 2.0/2.1. Aydin and others [8] took the application one step further and proposed an integrated system called the NFC Internal which takes advantage of NFC to guide users to their destination. Mobile phones used must be NFC enabled and also have an embedded indoor navigation application. Guerrero, Vasquez and Ochoa [10], took the studies done by Sonnenblick [11] who implemented a system based on infrared LEDs and turned them into an improved navigational system. In Sonnenblick's research were captured by an infrared receptor narrowing down the coverage of the environment since the receptor has to point directly to the LEDs. Guerrero et.al took out this limitation by installing infrared LEDs in a radial form around a white cane in order to increase visibility between the light source and the receptor. There are three types of navigation, outdoor, indoor, and one that combines the two. Such a system is Drishti, produced by Helal, Moore and Ran [12]. GPS already mentioned is used for outdoor navigation only, and is not available indoors because of the loss of the line of sight with the satellite (Non-Line-of-Sight: NLOS). What Drishti does is to use DGPS as its own outdoors location system and when the user enters to an indoor environment Drishti switches to a different location tracking using ultrasound pilots located at the four corners of the house ceiling. In addition two beacons are attached on top of the user's shoulders and there is also a wearable computer around the user's waist.

A special algorithm has been developed to calculate the user's exact coordinates. Limitations in this system are the "dead spots" because of the small number of ultrasound pilots used and that the study was two-dimensional with an average height taken under consideration, since the study was lacking the user's height data. A different type of indoor navigation system is called PERCEPT and consists of the PERCEPT Android client and the PERCEPT server. The whole PERCEPT client system is incorporated into a glove that consists of an Arduino Microcontroller, an RFID Reader Bluetooth chip and a Power Regulator. The glove has the ability to communicate through passive RFID tags and using Bluetooth technology to the Android based Smartphone. The tags are deployed on each door of the building. The Arduino microcontroller is used to keep track of all the events occurring during the interaction between the user wearing the PERCEPT glove and the environment. The Bluetooth chip is responsible for exchanging data between the microcontroller and the Android smartphone where a Wi-Fi module is used for establishing Wi-Fi connection between the Android Smartphone and the PERCEPT Server. At last the server returns to the user the navigational instructions needed over a Wi-Fi connection. A main disadvantage of this system is that the tags can accidentally get scanned or the buttons pressed by the user [13].

\section{CONCEPTUALIZATION}

\section{A. Localisation}

Setting the framework of decoding the blueprints of a building $B$ into a vector space via $G$, we are able to define the optimal path for any possible route across B. However, this does not add to the literature since there are various methods that hold such a property. To overcome the trivia of this problem excess consideration is required on the local "behavior" of indoor navigation algorithm, i.e. localization. To optimize the localization process, emulation of "ordinary" movement by a non-disabled person is needed. In particular, mimicking the various direction changes every time an "event" arises.

\section{B. Obstacles}

We define two main categories of obstacles "expected" and "unexpected", subcategorized to mobile and stable.We define as "expected" obstacles those with probability $\operatorname{Pr}()=$. to occur at a pre-specified moment on a pre-specified spot inside G. On the other hand, we define as "unexpected" obstacles those with probability $\operatorname{Pr}()<$.1 to occur at a prespecified moment on a pre-specified spot inside G.

Adding to the above definitions we subcategorize expected and unexpected obstacles into "mobile" \{obstacles with random velocity and direction \& "stable" \{obstacles with constant velocity and direction $\}$. Since by definition expected obstacles have probability equal to 1 , they can be considered as predefined spots inside G. Hence, the only random variable in our set is errors or unexpected events i.e. "mobile" obstacles. Another key aspect in indoor navigation is the predictability power, PE, which is negatively correlated with "mobile" obstacles. Therefore,

$\mathcal{P} \mathcal{E}^{*}\left(x_{1}, c_{1}, \ldots, x_{n}, c_{n}\right)=\sum_{i}^{n} c_{i}+\mathcal{P} \mathcal{E}\left(x_{1}, \ldots, x_{n},\right)$ which simplifies $E\left[\mathcal{P E}\left(x_{1, \ldots,} x_{n}\right)\right]$, where $E[]$ is the expected value of the predictability power.

$\mathbb{P} E$ is assumed to be an explicit function, $\mathbb{C}_{\mathbb{1}}$ are constant numbers or known entities and $\mathrm{x}_{1}$ are random variables with $f\left[x_{i}\right] \in \mathbb{E}$.

\section{SYSTEM ARCHITECTURE}

The proposed system is designed to meet all known objectives that may restrain such types of applications otherwise. One of the main requirements in navigationing indoors is the accuracy of the methodology and how this results in the outputs of the process. This is the reason why the proposed system adopts a precise technology such as RFID, and thus assumes that the floors are equipped with the required RFID tags. By establishing the above, it enables the system to provide the aim for an analytically continuous procedure of 
localization and navigation even when recalculation of paths is necessary.

Moreover, in order to keep the computational cost in low levels the system is designed under the concept of a clientserver with the client to be more active. As far as the scalability and the adaptivity to potential changes of our system, methodologies and technologies are taken into consideration that are not limited by the amount of users and the size of the navigation grid.

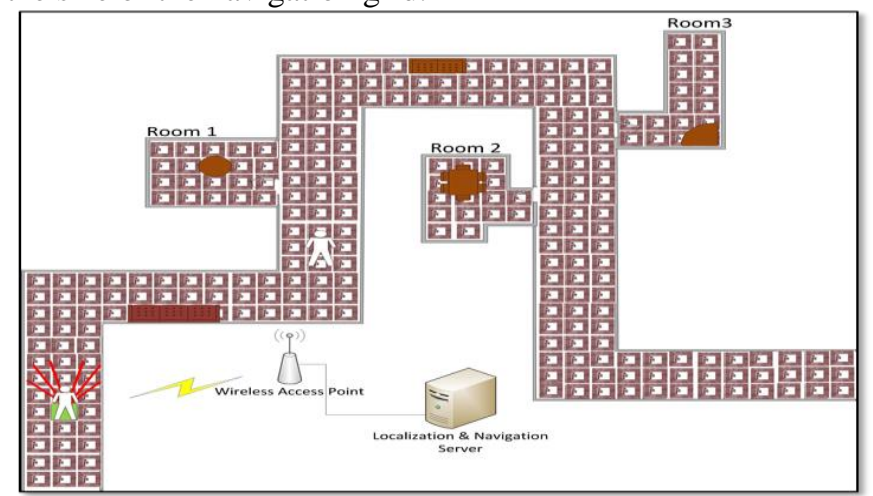

Figure 1 - Visualization of proposed system

All the above result in designing the system as illustrated in figure 3 in which the user can receive directions from a central server to be autonomous navigated to the desired final destination.

The proposed indoor navigation framework consists of 2 main components: a wearable module and a localization \& navigation server illustrated in figure 4 . Both modules run continuously and exchange information through a wireless access point.

The wearable module transfers the required data to the system enabling it to localize and navigate elderly people efficiently and in the best possible "natural" way. The module is composed of a microcontroller ATmega328 running the local processes, and is equipped with appropriate sensors and readers in order to collect all required data.

More specifically a RFID reader is emended in the wearable module, positioned on the leg of the user, capable of reading passive tags. It is designed for reading code from 125 $\mathrm{kHz}$ read and read/write tags. Additionally, it has a maximum effective reading distance for up to $50 \mathrm{~mm}$ and needs less than $100 \mathrm{~ms}$ for decoding the collected data.

A crucial part of the system's construction was the manufacturing of suitable RFID tags that would meet the requirements of our algorithm. This was feasible by assembling RFID tags with characteristics of the above designed antennas combined with a PIC microcontroller to emulate known RFID transmitting protocols such as EM4100 and EM4102.

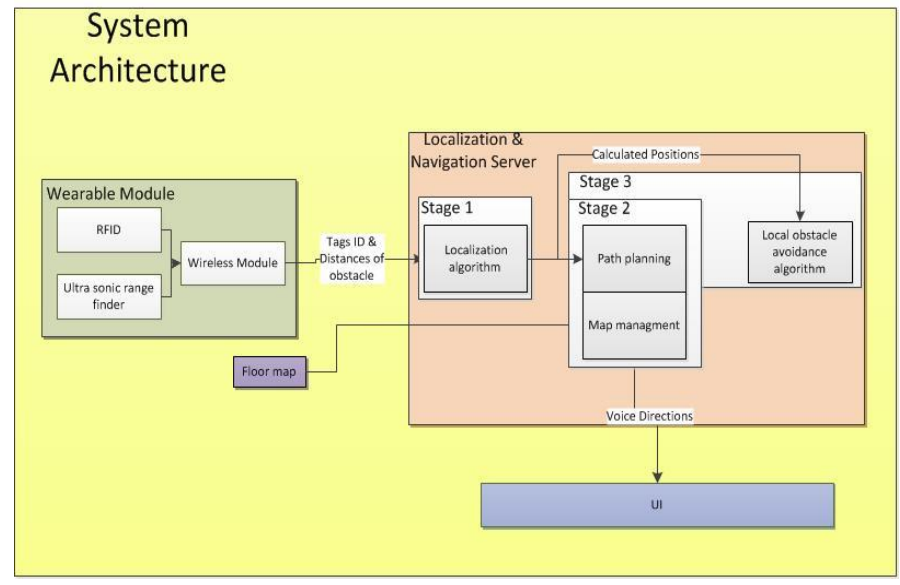

Figure 2-System Architecture

Furthermore an ultra-sonic range finder is additionally used to detect "leading" obstacles in the path ahead of the user. It measures a wide range from $3 \mathrm{~cm}$ to $6 \mathrm{~m}$, and the parameters of its range and gain are configurable variables that depend on the needs of our solution.

Another key part of the wearable module is the voice controller that is capable of playing up to $22 \mathrm{KHz}, 12$ bit uncompressed audio files of any length. Audio files are read off of an SD/MMC card and the volume can be controlled by an onboard thumbwheel potentiometer. At last, in need of exchanging data with the server a common Wi-Fi module is being used.

The other end of the system ends up to the server, which is responsible for the main operations of our framework grouped into three stages. In the $1^{\text {st }}$ stage the system initializes taking into account the blueprints of the floor map and the primary calculation of the shortest path. The $2^{\text {nd }}$ stage is a continuous process of the localization. Finally, the $3^{\mathrm{d}}$ stage, if needed, is undertaking the avoidance algorithm as well as the recalculation of the shortest path in case the user stray out of the navigation path.

\section{RESULTS}

In the testing process four blindfolded volunteers participated in different scenarios. For all of the scenarios the main scope was to localize and navigate a person in any given time successfully throughout an indoor environment in a natural way. The concept among scenarios was for the visually impaired person to be navigated around a floor through corridors avoiding expected and/or unexpected obstacles. The set of distances between the obstacles and the user that enables the person to move forward, to make a stop, or avoid the obstacles is a pre-configurable parameter. In this test the distances were fixed to: under $1.2 \mathrm{~m}$ ( 3 cells) for the stopping instruction, between $1.2 \mathrm{~m}$ and $2 \mathrm{~m}$ ( 5 cells) for the avoiding procedure, and above $2 \mathrm{~m}$ for continuing forward. The floor area that was used for the experiment was approximately 30 $\mathrm{m} 2$. The distribution of obstacles and the sequence of the scenarios were different for each volunteer, and every case was repeated numerous times.

The obtained results were the optimally visual outcomes of the system, since the scenarios took place in the exact same 
part of the route among all volunteers, for validation purposes. The size of the grid is $8 \times 8$ cells $(10.24 \mathrm{~m} 2)$ and the numbered point nodes constitute the center of each cell. The scenarios which took place are the following:

- The appearance of an unexpected obstacle that is observed towards the direction of the user and is always assumed to be the user's next step. The obstacle belongs in the set of avoidable distances $(1.2 \mathrm{~m}<\mathrm{d}<2 \mathrm{~m})$. The appearance of the unexpected obstacle in an avoidable distance and more specifically in $1.32 \mathrm{~m}$ triggers the system to calculate the avoidance path.

- The sudden appearance of an obstacle through the user's path, at a distance that obligates the user to make an emergency stop in order to avoid a collision, is the "worstcase" scenario event.

- The detection of an unexpected obstacle when the user is in the process of avoidance. In this situation the system alerts the user to come into an immediate stop.

The number of volunteers does not allow for a rigorous validation of the proposed system. However, during the testing additional metrics were captured to evaluate transversal aspects of the solution. In order to validate system's performance, we measured the time period between the highlight in which the user makes a movement and the moment in which the voice direction calls for a different movement to the user. Such a period was below $0.5 \mathrm{~s}$ in $99 \%$ of the cases. There was no difference if the user was on the move or immobile.

Additionally, evaluation was used to detect potential obstacles, to be classified as expected or unexpected and to provide the user with the best choice. In $99 \%$ of cases the system "exposed" the obstacles, in addition $99 \%$ of these cases were classified suitably and the user was instructed optimally. Moreover, other metrics associated to time needed to calculate in two phases the shortest path, localize at any given time and read a RFID tag distributed anywhere in the building are presented in table I.

TABLE I. TIME TAKEN AMONG PHASES

\begin{tabular}{|l|l|l|l|l|}
\hline Phases & $\begin{array}{l}\text { Calculate } \\
\text { Shortest } \\
\text { Path }\end{array}$ & $\begin{array}{l}\text { Recalculate } \\
\text { Shortest } \\
\text { Path }\end{array}$ & $\begin{array}{l}\text { Read } \\
\text { of } \\
\text { RFID } \\
\text { tag }\end{array}$ & Localization \\
\hline Time(sec) & 0.3 & 0.1 & 0.3 & 0.6 \\
\hline
\end{tabular}

\section{CONCLUSION}

In this paper a RFID-based architecture of an indoor navigation system, being able to navigate a visually impaired senior with safety in a natural way is presented. More specifically a "mapping" process by interpreting the blueprints of a building is proposed and an innovative localization and obstacle avoidance system based on the RFID technology was presented. All the above are integrated by developing the appropriate modules (wearable module and localization server) in a holistic indoor navigation framework that provides all the required features such as robust indoor localization, accurate navigation and efficient voice guidance. These features are tested in four blindfolded volunteers participating in various scenarios. Bearing in mind the time and human resource limitations, a 99\% success rate of our algorithm can't be considered as statistically significant. However, simulating different scenarios multiple times we safely assume the smoothness and predictive behavior of the algorithm

\section{REFERENCES}

[1] WHO Report:Visual impairment and blindness. 2013.

[2] Schiller, J.S., \& Peregoy, J.A. Provisional Report: Summary health statistics for U.S. adults:National Health Interview Survey. 2012. 10.

[3] Bradford, D. Detecting degeneration: Monitoring cognitive health in independent elders. in Engineering in Medicine and Biology Society (EMBC), 2013 35th Annual International Conference of the IEEE. 2013. IEEE.

[4] Kyriacou, E., et al., Multi-purpose HealthCare Telemedicine Systems with mobile communication link support. BioMedical Engineering OnLine, 2003. 2(7): p. 1-12.

[5] Lamprinos, I., et al. Energy-efficient MAC protocol for patient personal area networks. in Engineering in Medicine and Biology Society, 2005. IEEE-EMBS 2005. 27th Annual International Conference of the. 2006. IEEE.

[6] Istepanian, R., et al., Effect of wavelet compression on data transmission in a multipurpose wireless telemedicine system. Journal of telemedicine and telecare, 2000. 7: p. 14-16.

[7] Acharya, U., et al. Computer-based classification of eye diseases. in Engineering in Medicine and Biology Society, 2006. EMBS'06. 28th Annual International Conference of the IEEE. 2006. IEEE.

[8] Ozdenizci, B., et al. Development of an Indoor Navigation System Using NFC Technology. in Information and Computing (ICIC), 2011 Fourth International Conference on. 2011.

[9] Ivanov, R., Indoor navigation system for visually impaired, in Proceedings of the 11th International Conference on Computer Systems and Technologies and Workshop for PhD Students in Computing on International Conference on Computer Systems and Technologies. 2010, ACM: Sofia, Bulgaria. p. 143-149.

[10] Guerrero, L.A., F. Vasquez, and S.F. Ochoa, An indoor navigation system for the visually impaired. Sensors, 2012. 12(6): p. 8236-58.

[11] Sonnenblick, Y. An indoor navigation system for blind individuals. in Proceedings of the 13th annual Conference on Technology and Persons with Disabilities. 1998

[12] Ran, L., S. Helal, and S. Moore, Drishti: An Integrated Indoor/Outdoor Blind Navigation System and Service, in Proceedings of the Second IEEE International Conference on Pervasive Computing and Communications (PerCom'04). 2004, IEEE Computer Society. p. 23.

[13] Ganz, A., et al. PERCEPT: Indoor navigation for the blind and visually impaired. in Engineering in Medicine and Biology Society,EMBC, 2011 Annual International Conference of the IEEE. 2011. 